\title{
The Application of Marker Based Segmentation for Surface Texture Characterization
}

\author{
Nuraini binti Che $\operatorname{Pin}^{1, a}$, Mohd Fauzi bin Ismail ${ }^{1}$ and Siti Noraini binti Sulaiman ${ }^{2}$ \\ ${ }^{1}$ Faculty of Mechanical Engineering, University Technology Malaysia \\ ${ }^{2}$ Faculty of Electrical Engineering, University Technology Mara, Malaysia
}

\begin{abstract}
Structured surfaces have been increasingly used in industry for a variety of applications, including improving the tribological properties of the surfaces. Surface metrology plays an important role in this discipline since with the help of surface metrology technology, surface texture can be measured, visualize and quantified. Traditional surface texture parameters, such as roughness and waviness, cannot be related to the function for structured surfaces due to the less statistical description and little information. Therefore, a new approaches based on characterizing the structured surface is introduces where this paper focus on type of edges grain surface. To identify features, it is a must to detect the location of the edges and segmented the features based on the detected edges. Hence characterization of surface texture segmentation based on the edges detection is developing using Marker Based segmentation and it is prove that this method is possible to be used in order to characterize the structured surface.
\end{abstract}

\section{Introduction}

All surfaces whether at the nano or macro scale have made up of collection of fundamental features parameter which can be visualize in surface topography [1]. There are many type of surface of the product design but the new generation of surface nowadays is so called as structured surfaces. This type of surface has dominant deterministic pattern design to produce a specific function [2]. Besides, this kind of surface also has been increasingly used in industry for a variety of applications, including improving the tribological properties of the surfaces. High potential application of structures surfaces related product also found in the diverse automotive, biotech, medical and consumer application and are one of the current drivers of future growth.

Surface metrology plays an important role in this discipline since with the help of surface metrology technology, surface texture can be measured, visualize and quantified of their feature parameter on the surface. Over the years, the characterization and evaluation of engineering surface texture has constitutes a challenging metrological problem that has remained open so far, especially when high precision or structured surface with functional performance requirements exist. Besides that, it has been found that the standard areal parameters are often insufficient for characterizing structured surface, primarily because the areal surface texture parameters capture properties that are related to the entire measured topography whereas structured surface often exert their function through the action of individuals or multiple, localized surface features [3].

\footnotetext{
a Corresponding author: nuraini_chepin@yahoo.com
}

In this paper, a new approaches based on characterizing the structured surface is introduces. Initially it is useful to classify structured surface into two classes each based on their generic geometric form. This paper will focus on structured steps include surfaces that have grain, edges and facets [4]. Example of surface that has been tested is shown in Figure 1. This edges grain surface is characterize to extract the feature parameter such as the area, perimeter of the specific feature that have been selected on the surface. Consequently, this characterization will indirectly help the manufacturer to quantify the feature on the surface since in manufacturing process, if the measurement parameter is constant from any work piece to another work piece then the process is said to be under control.

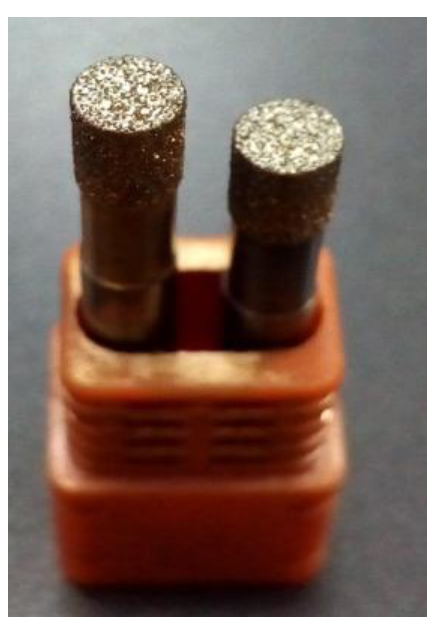

Figure 1. Sample of diamond grinding surface. 
To identify features on the structured surface, an important method required is to segment the surface into regions on interest [5]. Since this paper is focusing surface with edges features, therefore it is a must to detect the location of the edges and segmented the features based on the detected edges. Hence, this paper will develop the characterization of surface texture segmentation based on the edges detection method.

\section{Measurement method}

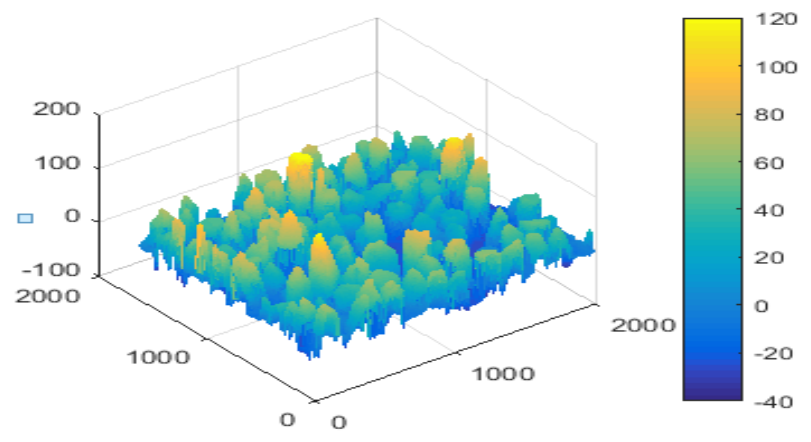

Figure 2. Topography surface of diamond grinding.

The confocal laser scanning is used to measure the surface of diamond grinding in the present study which shown in Figure 2. Surfaces are acquired point-by-point and reconstructed with a computer, allowing threedimensional reconstructions of topologically complex objects were obtained [6]. The surface topography profile gives true colour information that can be measured in $3 \mathrm{D}$ data. This data structure consist of a matrix in rectangular shape with regular $\mathrm{x}, \mathrm{y}$ and $\mathrm{z}$ grid where rows and columns referring to $\mathrm{x}$ and $\mathrm{y}$ while height referring to $\mathrm{z}$.

\section{Characterization method}

In order to characterize surface topography of the diamond grinding, the surface need to go preprocessing steps which are leveling and filtering. Function to do leveling to the surface is to provide a consistent reference plane so that it will become easier to characterize the whole surface later on. While filtering is done so that the outliers and noise of the measured data surface can be remove. This is due to the fact that measured data always have some irrelevant information that is not interested in.

Morphological filter is used in this research for the filtration process. This type of filtration is choosing with the fact that it can help to preserve the edges features on the surface [7]. Specific morphological filtration is used which are opening and closing by reconstruction. Reconstruction by opening and closing are more effective than standard opening and closing at removing small outlier without affecting the overall shapes of the surface. Disk is the structuring element that has been used with appropriate radius. Experimental testing is done to determine the best radius of disk to be used.

\subsection{Detection of significant edges}

Since this research study focus on edges segmentation, therefore a critical step is to detect the significant edges on the surface. Sobel Operator is the method of edges detection approaches that have been used to detect the significant edges. The size of this kernel of operator is set to be fixed with $3 \times 3$ size of kernel.

\subsection{Creating marker controlled based}

In this research, simple method is used to create the foreground and background marker which is by using morphological reconstruction. In morphological reconstruction, structuring element used was playing an important role since it gives big impact to the number of segmented features later on. Two steps involved in this steps which are erosion based reconstruction and followed by dilation based reconstruction. Regional maxima also being calculated in order to get smooth edge foreground objects. Later on, these two steps and the edges detection surface are been superimposed on the surface that has been filtered [8].

\subsection{Segmentation using watershed}

Application of watershed segmentation will gives final segmentation results of the surface. Therefore, full algorithm of marker based segmentation is shown on the flowchart in Figure 3.

\begin{tabular}{|c|}
\hline Develop edges \\
detection \\
surface \\
using Sobel \\
operator \\
algorithm \\
\hline
\end{tabular}
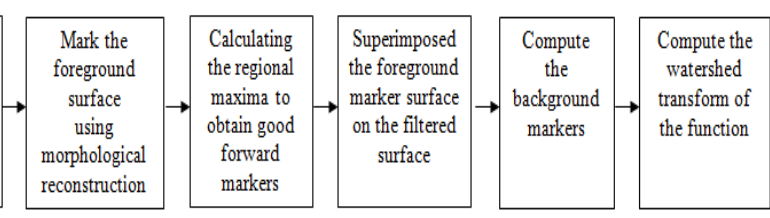

Figure 3. Algorithm for maker based segmentation.

\section{Discussion}

In order to get better segmentation results, radius of disk in morphological filtration need to choose correctly. Different radius of disk is tested to see the effect of the segmentation results. Radius of the disk is tested starting from $1 \mu \mathrm{m}, 3 \mu \mathrm{m}, 5 \mu \mathrm{m}, 7 \mu \mathrm{m}$ and finally $9 \mu \mathrm{m}$. The graph in Figure 4 shows the results from the testing.

Based on the graph in Figure 4, it shows that the number of segmentation is decrease accordingly with the increase of radius of the structuring element until one point that the number of segmentation becomes more stabilized. Besides, it can be compared that the count of number of segmentation features at $1 \mu \mathrm{m}$ radius of disk, the number of segmentation has sharply reducing at $3 \mu \mathrm{m}$ radius. Then the count becomes linear after $5 \mu \mathrm{m}$ of radius of the disk.

\section{Marker based segmentation}




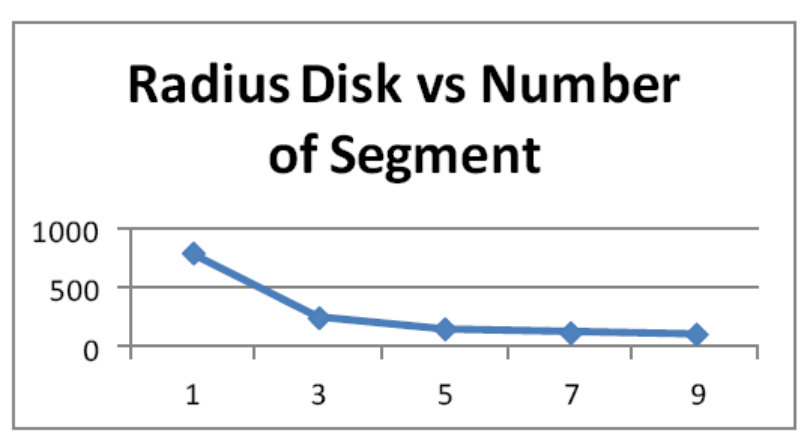

Figure 4. Graph of number of features segmentation versus radius of structuring element.

To make it more clear, Figure 5 shows the view from the top of the segmentation results after undergo Marker Based Segmentation. When radius of the structuring element used was too small, over segmentation is happen to the surface. While after radius of the structuring element is increase, number of feature segmentation become more stable and linear.

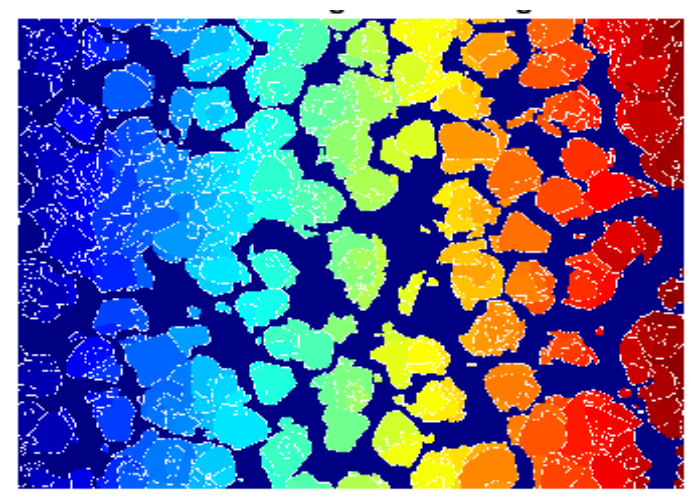

800 number of features

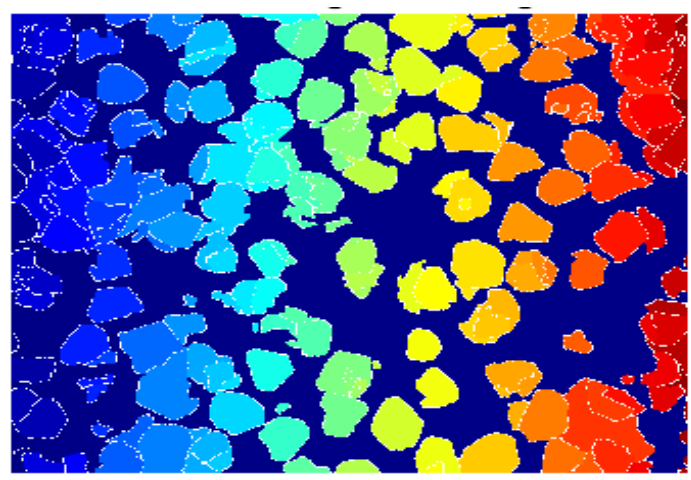

254 number of features

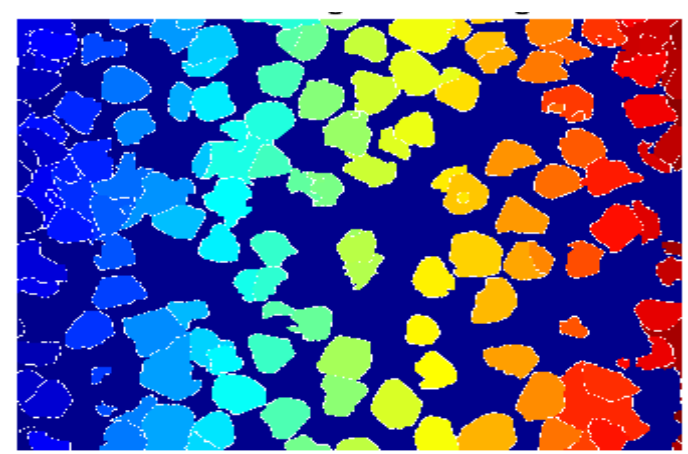

160 number of features

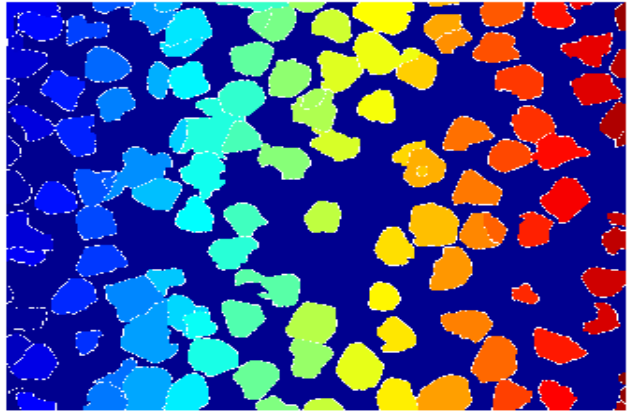

115 number of features

Figure 5. Different number of features segmentation based on different radius of disk.

\section{Conclusion}

As the conclusion, Marker Based Segmentation is possible to be used to detect the edges on the surface data topography and finally segment the surfaces following the shaped of the features. Morphological Filtration, Sobel Operator, Regional Maxima and also Watershed Segmentation are the best method to be paired in group for the overall algorithm of the Marker Based Segmentation. For further work, all the related feature parameter such as area, perimeter, volume and others will be specifically identified.

\section{Acknowledgement}

The authors gratefully acknowledge University Technology MARA and Ministry of Higher Education Malaysia for the financial support under Fundamental Research Scheme (Grant no: FRGS/1/2014/TK01/UITM/02/6).

\section{References}

1. L. Blunt and S. Xiao, "The use of surface segmentation methods to characterise laser zone surface structure on hard disc drives," Wear, 271, 34 604-609 (2011)

2. G. D. MacAulay, N. Senin, C. L. Giusca, R. K. Leach, and A. Ivanov, "Review of feature boundary identification techniques for the characterization of tessellated surfaces," Surf. Topogr. Metrol. Prop., 3, 1013002 (2015)

3. G. D. Macaulay, N. Senin, C. L. Giusca, and R. K. Leach, "Comparison of Segmentation Techniques to Determine the Geometric Parameters of Structured Surfaces," Surf. Topogr. Metrol. Prop., (2014)

4. X. Jiang, P. Scott, and D. Whitehouse, "Freeform Surface Characterisation - A Fresh Strategy," CIRP Ann. - Manuf. Technol., 56, 1 553-556 (2007)

5. A. G. Demir, P. Maressa, and B. Previtali, "Fibre laser texturing for surface functionalization," Phys. Procedia, 41, 759-768 (2013)

6. J. Peters, J. B. Bryan, W. T. Estler, C. Evans, H. Kunzmann, D. a. Lucca, S. Sartori, H. Sato, E. G. Thwaite, P. Vanherck, R. J. Hocken, J. Peklenik, T. 
Pfeifer, H. Trumpold, and T. V. Vorburger, "Contribution of CIRP to the Development of Metrology and Surface Quality Evaluation during the last fifty years," CIRP Ann. - Manuf. Technol., 50, 2 471-488 (2001)

7. P. Dobrzaski and P. Pawlus, "A study of filtering techniques for areal surface topography assessment,"
Proc. Inst. Mech. Eng. Part B J. Eng. Manuf., 225, 11 2096-2107 (2011)

8. R. Maini and J. S. Sohal, "Performance Evaluation of Prewitt Edge Detector for Noisy Images," Int. J. Graph. Vis. Image Process., 6, 3 39-46 (2006) 